Enhanced magnetoresistance and Griffiths phase induced by Mo substitution in $\mathrm{La}_{0.7} \mathrm{Ca}_{0.15} \mathrm{Sr}_{0.15} \mathrm{Mn}_{1-x} \mathrm{Mo}_{x} \mathrm{O}_{3}(0 \leq x \leq 0.05)$

This article has been downloaded from IOPscience. Please scroll down to see the full text article. 2009 J. Phys. D: Appl. Phys. 42095003

(http://iopscience.iop.org/0022-3727/42/9/095003)

The Table of Contents and more related content is available

Download details:

IP Address: 140.112.113.225

The article was downloaded on 30/06/2009 at 09:07

Please note that terms and conditions apply. 


\title{
Enhanced magnetoresistance and Griffiths phase induced by Mo substitution in $\mathrm{La}_{0.7} \mathrm{Ca}_{0.15} \mathrm{Sr}_{0.15} \mathrm{Mn}_{1-x} \mathrm{Mo}_{x} \mathrm{O}_{3}$ $(\mathbf{0} \leqslant \boldsymbol{x} \leqslant \mathbf{0 . 0 5})$
}

\author{
G Narsinga Rao ${ }^{1}$, J W Chen ${ }^{1,3}$, S Neeleshwar ${ }^{2,4}$, Y Y Chen ${ }^{2}$ and M K Wu ${ }^{2}$ \\ ${ }^{1}$ Department of Physics, National Taiwan University, Taipei, Taiwan, Republic of China \\ ${ }^{2}$ Institute of Physics, Academia Sinica, Taipei, Taiwan, Republic of China \\ E-mail: jwchen@phys.ntu.edu.tw (J W Chen)
}

Received 12 November 2008, in final form 4 March 2009

Published 16 April 2009

Online at stacks.iop.org/JPhysD/42/095003

\begin{abstract}
Structural, magnetic and electrical properties of the $\mathrm{La}_{0.7} \mathrm{Ca}_{0.15} \mathrm{Sr}_{0.15} \mathrm{Mn}_{1-x} \mathrm{Mo}_{x} \mathrm{O}_{3}$ $(0 \leqslant x \leqslant 0.05)$ compounds have been investigated. Powder $\mathrm{x}$-ray analysis reveals that the sample with $x=0$ crystallizes in the rhombohedral $(R \overline{3} c)$ structure, whereas in the Mo doped samples the structure can be indexed by an orthorhombic (Pbnm) structure. The important observations of the magnetic and transport properties are: (i) the Mo substitution induces a distinct suppression of the metal-insulator $\left(T_{\mathrm{MI}}\right)$ and ferromagnetic $(\mathrm{FM})$ - paramagnetic transition $\left(T_{\mathrm{C}}\right)$ and the temperature of $T_{\mathrm{MI}}$ was found to be higher than $T_{\mathrm{C}}$ in the Mo-doped samples, (ii) the substitution of Mo enhances the magnetoresistance at room temperature, (iii) a large deviation from the Curie-Weiss law well above $T_{\mathrm{C}}$ in the Mo substituted samples indicates the existence of a Griffiths phase and (iv) long-range FM order persists in all samples with a linear decrease of saturation moment as $x$ increases. These results are discussed in terms of the Mn-site disorder and opening of strong FM coupling between $\mathrm{Mn}^{2+}-\mathrm{O}-\mathrm{Mn}^{3+}$, due to the $\mathrm{Mn}^{2+}$ ions induced by $\mathrm{Mo}^{6+}$ at the expense of $\mathrm{Mn}^{4+}$ ions in the $\mathrm{La}_{0.7} \mathrm{Ca}_{0.15} \mathrm{Sr}_{0.15} \mathrm{Mn}_{1-x} \mathrm{Mo}_{x} \mathrm{O}_{3}$ system.
\end{abstract}

(Some figures in this article are in colour only in the electronic version)

\section{Introduction}

The phenomenon of colossal magnetoresistance (CMR) in mixed manganites $R_{1-x} A_{x} \mathrm{MnO}_{3}$ ( $R$ is a trivalent rare-earth ion and $A$ is a divalent alkaline-earth metal ion) have attracted considerable attention in recent years [1-3]. The CMR effect is initially explained within the framework of the Zener double exchange (DE) mechanism [4]. Additional effects, e.g. the strong Jahn-Teller (JT) interactions and phase separation (PS) are reported to play important roles

3 Author to whom any correspondence should be addressed.

4 Present address: School of Basic Sciences, GGS Indraprastha University, Delhi, India. in these materials $[5,6]$. Recent studies reveal that the CMR system is intrinsically inhomogeneous even in the best crystals, where the competition between the ferromagnetic metallic (FMM) and the paramagnetic insulating (PMI) phases results in the electronic PS [7-9]. It was observed that the existence of ferromagnetic (FM) clusters in paramagnetic (PM) phase is well above the Curie temperature, $T_{\mathrm{C}}$. Due to the inhomogeneous distribution of FM clusters, the existence of Griffiths [10] temperature $T_{\mathrm{G}}$ above $T_{\mathrm{C}}$ has been predicted in a number of perovskite manganites. The phase in the temperature range between $T_{\mathrm{G}}$ and $T_{\mathrm{C}}$ is usually referred to as the Griffiths phase (GP) [11]. Such a system exhibits a sharp downturn in the inverse of high temperature susceptibility 
above $T_{\mathrm{C}}$. In several reports, it is argued that the CMR effect should be treated in the context of a Griffith's singularity driven by intrinsic randomness, the combined effect of doping, the tendency for charge segregation and the self-trapping effect associated with polaron formation [9, 12-17]. Tong et al [18] indicated that the GP is not necessary to be tied with the CMR in the electron doped manganites. They indicated that the strain field, in addition to quenched disorders, may be an alternative approach to understand the observed GP. Jiang et al [19] also reported that the GP account for CMR is not a prerequisite in manganites. However, the GP in manganites linked to CMR is the subject of ongoing discussion and current attention.

The nature of local magnetic coupling between the neighbouring spins can only be addressed by $\mathrm{Mn}$ site substitution. This is an effective trigger for PS and to exhibit enormous magnetoresistance (MR). The results of such substitution depend on the reference state and the chemical nature of the impurity. Different effects can be produced upon Mn-site doping in FM manganites. There have been a great number of reports on the substitution effects of $\mathrm{Mn}$ cations by non-magnetic ions such as $\mathrm{Al}, \mathrm{Ti}, \mathrm{Ga}, \mathrm{Sc}$ and $\mathrm{Ta}$ [20-25]. It has been found that doping in Mn sites with foreign elements in general destroys DE ferromagnetism in the samples, which is caused by decreasing the $T_{\mathrm{C}}$ and $T_{\mathrm{MI}}$ temperatures dramatically. More interestingly, the PM unfilled $e_{\mathrm{g}}$ orbital ( $\mathrm{Ru}, \mathrm{Cr}, \mathrm{Co}, \mathrm{Cu}, \mathrm{Fe}$ and $\mathrm{Ni}$ ) [26-32] ions at the $\mathrm{Mn}$ site in manganites cause a PS. Magnetic dopants like $\mathrm{Cr}$, Co or $\mathrm{Ni}$ on the $\mathrm{Mn}$ site in charge ordered insulating manganites promote a percolative FM metal, while non-magnetic dopants of the same valence do not [33-35]. Both magnetic and non-magnetic dopants destabilized orbital ordered A-type antiferromagnet in favour of a FM state [36]. However, the Mo substitution at the Mn site destroys the antiferromagnetism (AFM) and FM state prevailing in A or G-type AFM insulators $[37,38]$. Such FM ordering is due to the different Mn species $\left(\mathrm{Mn}^{2+}\right.$ and $\left.\mathrm{Mn}^{3+}\right)$ induced by $\mathrm{Mo}^{6+}$. Nevertheless, Mo-doped $\mathrm{La}_{0.67} \mathrm{Ba}_{0.33} \mathrm{Mn}_{1-x} \mathrm{Mo}_{x} \mathrm{O}_{3}$ compounds exhibit the magnetic pair formation between $\mathrm{Mn}^{3+}: \mathrm{Mo}^{6+} \Leftrightarrow \mathrm{Mn}^{4+}: \mathrm{Mo}^{4+}$ [39]. Recently, the mixed valence state of $\mathrm{Mn}\left(\mathrm{Mn}^{2+}, \mathrm{Mn}^{3+}\right.$ and $\mathrm{Mn}^{4+}$ ) co-existing in $\mathrm{La}_{0.7} \mathrm{Ca}_{0.3} \mathrm{Mn}_{1-x} \mathrm{Mo}_{x} \mathrm{O}_{3}$ compounds by partial substitution of $\mathrm{Mo}^{6+}$ were reported [40] since Mo can exist with different valence states and induces different $\mathrm{Mn}$ valences.

$\mathrm{La}_{0.7} \mathrm{~A}_{0.3} \mathrm{MnO}_{3}(A=\mathrm{Ca}$ or $\mathrm{Sr})$ compounds are studied carefully to optimize the Curie temperature and MR. The phase diagram of these compounds ( $T_{\mathrm{C}}$ dependence on $\left.\left\langle r_{\mathrm{A}}\right\rangle\right)$ is well known and appears like a bell shape [41]. In particular, if $A=\mathrm{Ca}$, then the value $\mathrm{MR}$ is maximum with lower $T_{\mathrm{C}}$. But in the $\mathrm{Sr}$ case, the $T_{\mathrm{C}}$ is higher with low MR value. Interestingly, the $\mathrm{La}_{0.7} \mathrm{Ca}_{0.3-x} \mathrm{Sr}_{x} \mathrm{MnO}_{3}$ system undergoes a structural transition between the orthorhombic $(\mathrm{Pbnm})$ and the rhombohedral $(R \overline{3} c)$ structure depending on the variation of both $x$ and the temperature. The average ionic radius $r_{\mathrm{A}}=$ $1.227 \AA$ corresponding to $x=0.15$ exhibits the concentration structural phase transition [42-45]. Moreover, this transition is controlled by an external magnetic field, indicating a small difference between the ground state energies for these crystal symmetries [43]. Therefore, it is reasonable to suggest that any external perturbation of the crystal lattice (such as lattice strain) leads to a structural modification in this compound. It is worthwhile to investigate the influence of the substitution at the Mn site by other elements without disturbing $r_{\mathrm{A}}$ in the vicinity of the above noted structural and FM phase transition.

Among all Mn-site substitutions, the Mo substitution effect has been considered to be of particular interest, due to its high valence state $\left(\mathrm{Mo}^{6+}\right)$ and its $\mathrm{d}^{0}$ electronic configuration. Even the small amount of Mo doping is anticipated to induce a large variation in the $\mathrm{Mn}$ valence. Moreover the effect of Mn-site substitution in $\mathrm{La}_{0.7} \mathrm{Ca}_{0.15} \mathrm{Sr}_{0.15} \mathrm{MnO}_{3}$ compound has never been reported as we know. In this paper, we study the effect of Mo substitution on structural, magnetic and transport properties of the $\mathrm{La}_{0.7} \mathrm{Ca}_{0.15} \mathrm{Sr}_{0.15} \mathrm{Mn}_{1-x} \mathrm{Mo}_{x} \mathrm{O}_{3}$ system. This system shows interesting GP in Mo-doped samples.

\section{Experimental details}

Polycrystalline samples of nominal composition $\mathrm{La}_{0.7} \mathrm{Ca}_{0.15}$ $\mathrm{Sr}_{0.15} \mathrm{Mn}_{1-x} \mathrm{Mo}_{x} \mathrm{O}_{3} \quad(x=0$ to 0.05 with steps of 0.01 ) were synthesized by the solid-state reaction method. Stoichiometric proportions of dried high purity $\mathrm{La}_{2} \mathrm{O}_{3}$ (99.999\%), $\quad \mathrm{CaCO}_{3}$ (99.9\%), $\quad \mathrm{SrCO}_{3}$ (99.9\%), $\quad \mathrm{MnCO}_{3}$ (99.99\%) and $\mathrm{MoO}_{3}(99.99 \%)$ powders were mixed and first fired in the air at $900{ }^{\circ} \mathrm{C}$ for $12 \mathrm{~h}$. The pre-heated powders were ground thoroughly and calcined at $1200^{\circ} \mathrm{C}$ for $12 \mathrm{~h}$ with intermediate grinding. The calcined powders were then pressed into pellets and sintered at $1400^{\circ} \mathrm{C}$ for $24 \mathrm{~h}$. All the samples were prepared under identical conditions. The structure and phase purity of the samples were checked by powder $\mathrm{X}$-ray diffraction (XRD) using the $\mathrm{Cu}-\mathrm{K} \alpha$ radiation at room temperature. The field cooled (FC) and zero field cooled (ZFC) magnetization curves were measured in a commercial SQUID magnetometer with the temperature range from $4 \mathrm{~K}$ to $400 \mathrm{~K}$ in an applied field of 50 and $1 \mathrm{kOe}$, and the hysteresis curves were measured at $10 \mathrm{~K}$ for $-50 \mathrm{kOe} \leqslant H \leqslant+50 \mathrm{kOe}$. The dc resistivity and MR of the samples were measured using the conventional four-probe method in the temperature range from 10 to $350 \mathrm{~K}$ in a closed cycle helium refrigerator placed in a water cooled electromagnet with a field from $-8.9 \mathrm{kOe}$ to $+8.9 \mathrm{kOe}$. The MR ratio of the samples is defined as $\mathrm{MR}=[\rho(H)-\rho(0)] / \rho(0)$, with $\rho(0)$ and $\rho(H)$ being the resistivity measured at zero magnetic field and an applied magnetic field $H$, respectively.

\section{Results and discussion}

The XRD patterns of the polycrystalline $\mathrm{La}_{0.7} \mathrm{Ca}_{0.15} \mathrm{Sr}_{0.15}$ $\mathrm{Mn}_{1-x} \mathrm{Mo}_{x} \mathrm{O}_{3}$ samples are shown in figure 1(a). The XRD pattern of the sample $x=0$ can be categorized by a rhombohedral structure with space group $R \overline{3} c$. On the other hand, the Mo doped samples change structure and it was indexed by an orthorhombic (Pbnm) structure. The enlarged view of the $2 \theta=32-33.5^{\circ}$ (figure $1(b)$ ) range illustrates how the intense reflections of the $R \overline{3} c$ doublet (1 10 and 104) transform into the 121 reflection of the Pbnm phase with weaker 002 reflection when the $\mathrm{Mn}$ is partially substituted by Mo. The phase transformation can be clearly shown in 

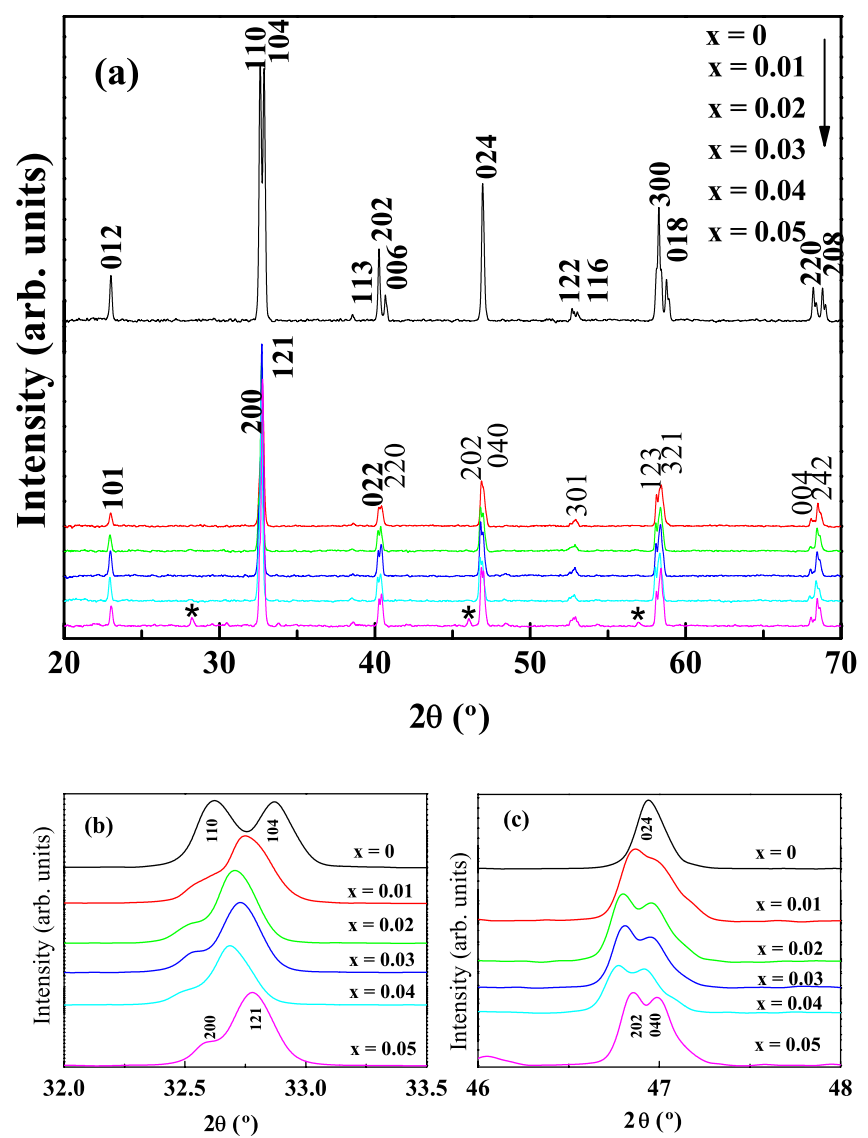

Figure 1. (a) The XRD patterns of $\mathrm{La}_{0.7} \mathrm{Ca}_{0.15} \mathrm{Sr}_{0.15} \mathrm{Mn}_{1-x} \mathrm{Mo}_{x} \mathrm{O}_{3}$ samples, the miller indices of the major Bragg reflections are indicated and the secondary phase marked as an asterisk, $(b)$ the enlarged view of the $2 \theta=32-33.5^{\circ}$ and $(c)$ the enlarged view of the $2 \theta=46-48^{\circ}$.

the enlarged view of the $2 \theta=46-48^{\circ}$ (figure $1(c)$ ) range, the intense 024 reflection of the $R \overline{3} c$ phase splits into 202 and 040 reflections of Pbnm. The patterns clearly reflect the structural changes caused by the replacement of Mn by Mo. In higher Mo-doped samples, a small secondary phase of $\mathrm{Sr}_{0.5} \mathrm{Ca}_{0.5} \mathrm{MoO}_{4}$ [46] was detected and it is marked by an asterisk in figure $1(a)$. The structural parameters of the samples are refined by the standard Rietveld technique. Typical XRD patterns along with Rietveld refinement are shown in figure 2 for the $(a) x=0$ and $(b) x=0.03$. It shows that the fitting between the experimental spectra and the calculated values is relatively good. The lattice parameters increase monotonically with increasing Mo content (figure $2(c)$ ). The most stable valence state of Mo is $6+$, which converts the $\mathrm{Mn}^{4+}$ into $\mathrm{Mn}^{2+}$ ions in order to keep the charges balanced. The increase in the lattice parameters is consistent with the increasing concentration of $\mathrm{Mn}^{2+}(0.83 \AA)$, which is larger than $\mathrm{Mn}^{3+}(0.645 \AA)$ or $\mathrm{Mn}^{4+}(0.54 \AA)$ ion [47]. A decrease in the lattice parameter for the sample with $x=0.05$ is due to the existence of a small secondary phase.

The variation of magnetization $(M)$ as a function of temperature for the $\mathrm{La}_{0.7} \mathrm{Ca}_{0.15} \mathrm{Sr}_{0.15} \mathrm{Mn}_{1-x} \mathrm{Mo}_{x} \mathrm{O}_{3}$ samples in a field of $H=1 \mathrm{kOe}$ is shown in figure 3. The nearly temperature independent behaviour observed in the $M(T)$ curves at low temperature indicates that the long range FM
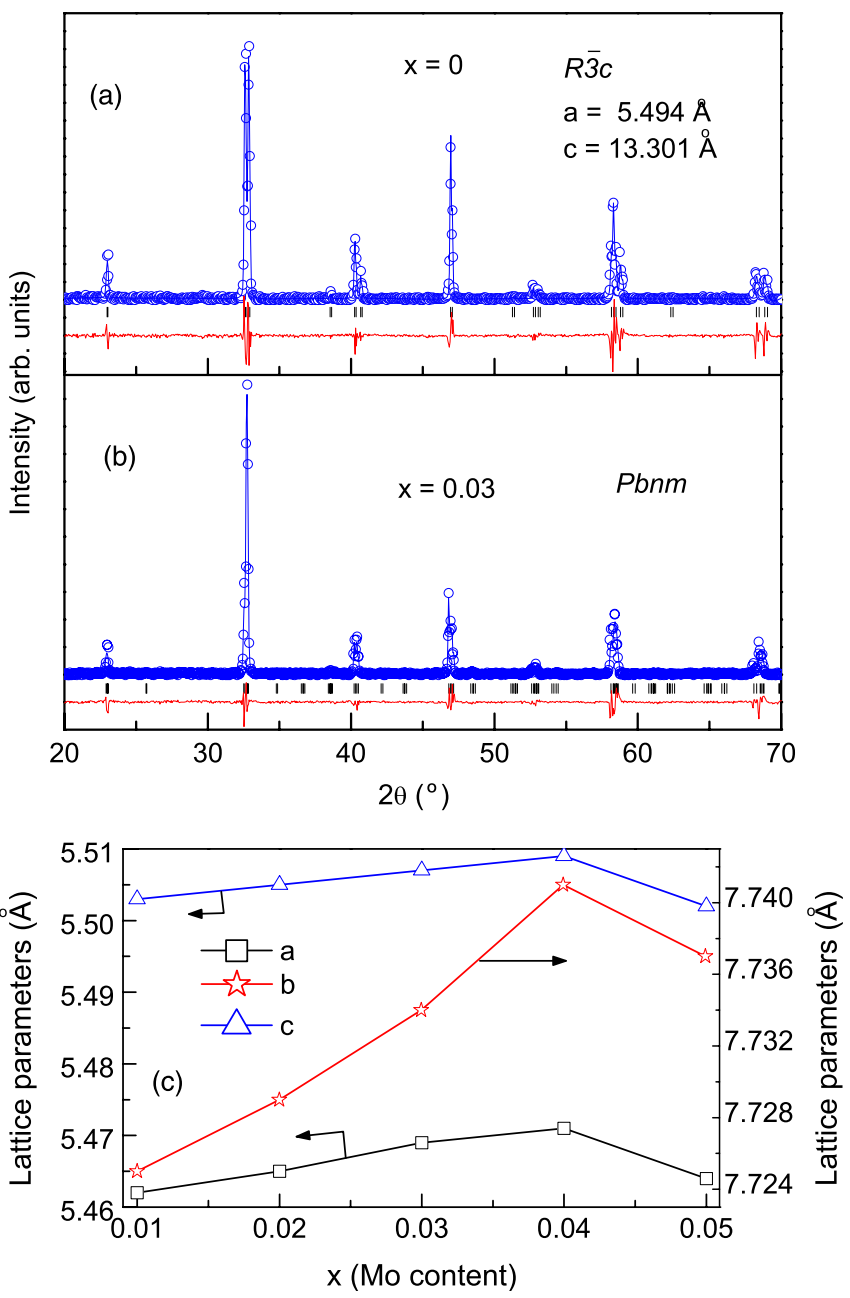

Figure 2. Rietveld refinement spectra of $\mathrm{La}_{0.7} \mathrm{Ca}_{0.15} \mathrm{Sr}_{0.15} \mathrm{Mn}_{1-x} \mathrm{Mo}_{x}$ $\mathrm{O}_{3}$ samples where $(a) x=0$ and $(b) x=0.03$. In each spectrum, the symbol denotes the observed intensity $\left(I_{\mathrm{obs}}\right)$, and the continuous line denotes the calculated intensity $\left(I_{\text {cal }}\right)$. The difference between the observed and the calculated intensities is shown at the bottom of the figure. The calculated Bragg-reflected positions are marked by the vertical bars. $(c)$ The variation of lattice parameters as a function of $x$.

ordering persists for all samples. A sharp transition from a PM to a FM state is observed for all samples. For the undoped sample, the obtained value of the $T_{\mathrm{C}}$ is $\sim 330 \mathrm{~K}$, consistent with previous reports [43]. The $T_{\mathrm{C}}$ is defined as the temperature corresponding to the maximum of $-\mathrm{d} M / \mathrm{d} T$ in the $M(T)$ curve. It is observed that the $T_{\mathrm{C}}$ decreases monotonically with increasing $x$, where $T_{\mathrm{C}}$ of $\sim 292 \mathrm{~K}$ is observed for the sample with $x=0.05$. The suppression rate $\left(\mathrm{d} T_{\mathrm{C}} / \mathrm{d} x\right)$ is found to be $7.6 \mathrm{Kat} \%^{-1}$, which is much smaller than that of the other $\mathrm{Mn}$-site doped systems. This indicates the existence of strong magnetic coupling between the neighboring Mn ions. Further evidence can be obtained from the low temperature hysteresis curves.

Figure 4 shows the field dependence of magnetization for the $\mathrm{La}_{0.7} \mathrm{Ca}_{0.15} \mathrm{Sr}_{0.15} \mathrm{Mn}_{1-x} \mathrm{Mo}_{x} \mathrm{O}_{3}$ samples at $10 \mathrm{~K}$. The magnetization tends to saturate at low fields for all the samples, indicating a long-range FM order. The saturation magnetic moment for $\mathrm{Mn}$ ion $\left(\mu_{\exp }\right)$ obtained at $5 \mathrm{~T}$ decreases linearly with increasing $x$, as shown in the inset of figure 4 . Considering 


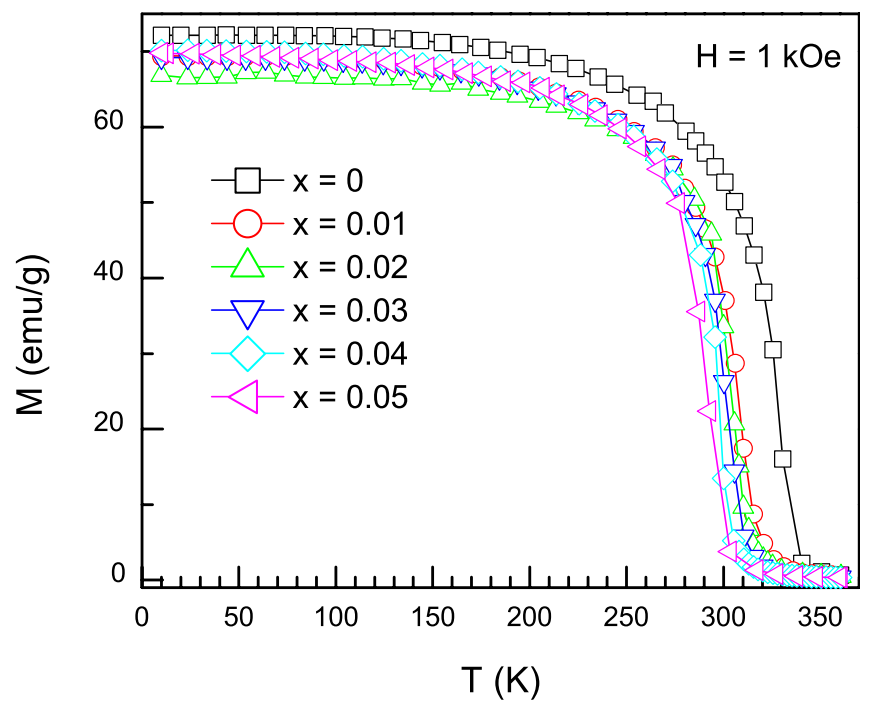

Figure 3. The variation of magnetization $(M)$ as a function of temperature for $\mathrm{La}_{0.7} \mathrm{Ca}_{0.15} \mathrm{Sr}_{0.15} \mathrm{Mn}_{1-x} \mathrm{Mo}_{x} \mathrm{O}_{3}$ samples measured in a field of $1 \mathrm{kOe}$.

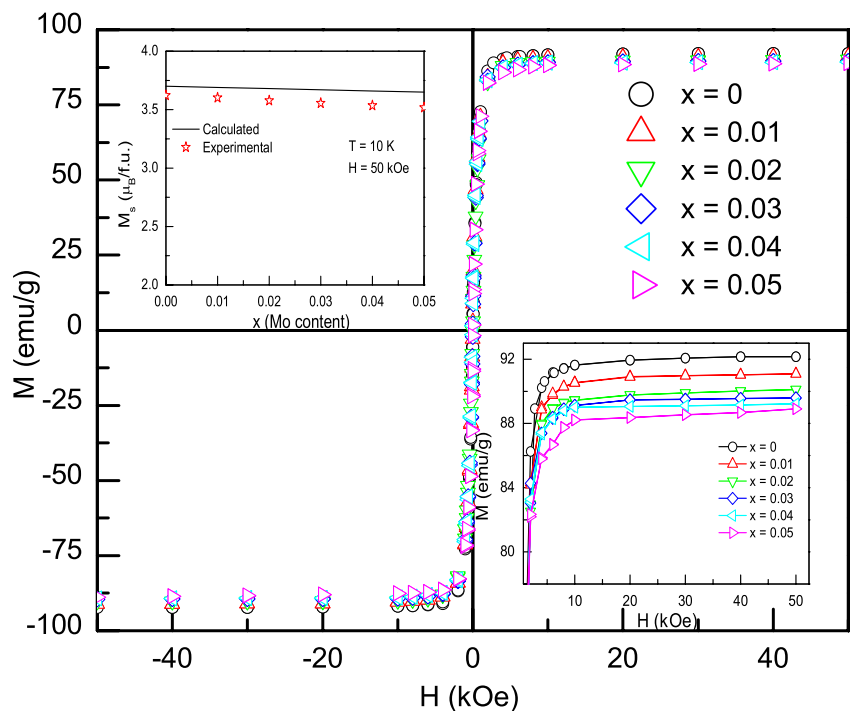

Figure 4. The isothermal $M(H)$ curves for $\mathrm{La}_{0.7} \mathrm{Ca}_{0.15} \mathrm{Sr}_{0.15} \mathrm{Mn}_{1-x}$ $\mathrm{Mo}_{x} \mathrm{O}_{3}$ samples measured at $10 \mathrm{~K}$ for $-50 \mathrm{kOe} \leqslant H \leqslant 50 \mathrm{kOe}$. The right inset shows the expanded view and the left inset shows the experimental and calculated saturated magnetic moment per formula unit as a function of $x$.

the dilution of non-magnetic $\mathrm{Mo}^{6+}$, the moment per $\mathrm{Mn}$ ion is expected to be $(1-x) \mu_{\text {exp }}$, but the value of $\mu_{\text {exp }}$ is higher for the doped samples. This indicates that the substitution of Mo does not merely destroy DE ferromagnetism, since, a much higher decreasing rate in $\mathrm{d} T_{\mathrm{C}} / \mathrm{d} x$ and $\mu_{\text {exp }}$ should obtain.

The partial substitution of hexavalent $\mathrm{Mo}^{6+}$ not only decreases the $\mathrm{Mn}^{4+}$ concentration but also induces the $\mathrm{Mn}^{2+}$ ions according to the charge neutrality formula $\mathrm{La}_{0.7}^{3+} \mathrm{Ca}_{0.15}^{2+} \mathrm{Sr}_{0.15}^{2+} \mathrm{Mn}_{0.7}^{3+} \mathrm{Mn}_{0.3-2 x}^{4+} \mathrm{Mn}_{x}^{2+} \mathrm{Mo}_{x}^{6+} \mathrm{O}_{3}$. Similar divalent $\mathrm{Mn}$ spectral features as those reported in this work were recently reported in $\mathrm{La}_{0.7} \mathrm{Ca}_{0.3} \mathrm{Mn}_{1-x} \mathrm{Mo}_{x} \mathrm{O}_{3}$ system [40]. The amount of $\mathrm{Mn}^{2+}$ will increase with increasing Mo content giving rise to an increase in $d_{\mathrm{Mn}-\mathrm{O}}$ and a decrease in $\theta_{\mathrm{Mn}-\mathrm{O}-\mathrm{Mn}}$. Thus, the DE interaction weakens because of the narrowing

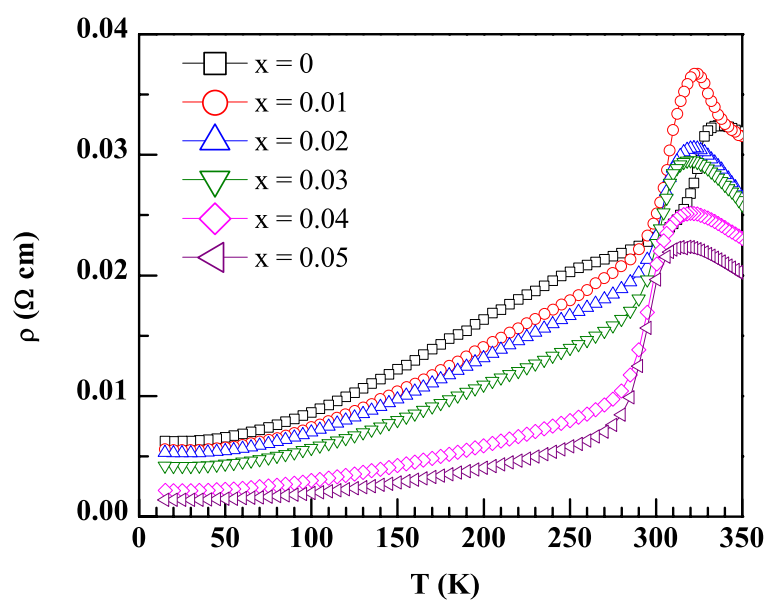

Figure 5. The temperature dependence of resistivity $\rho(T)$ curves for $\mathrm{La}_{0.7} \mathrm{Ca}_{0.15} \mathrm{Sr}_{0.15} \mathrm{Mn}_{1-x} \mathrm{Mo}_{x} \mathrm{O}_{3}$ samples measured at zero field.

of the bandwidth and $T_{\mathrm{C}}$ would decrease with the increase in the Mo-doping level. This formula is consistent with the XRD measurements. But the lattice parameters for $x=0.05$ are quite different, not following the trend displayed by other concentrations. Surprisingly, the magnetic properties of $x=0.05$ are not special. The observed impurity phase $\mathrm{Sr}_{0.5} \mathrm{Ca}_{0.5} \mathrm{MoO}_{4}$ is a non-magnetic compound, due to empty $4 \mathrm{~d}$ orbits in $\mathrm{Mo}^{6+}$ ions. Because of this impurity phase there is a small deficiency in the A site (i.e. $2+$ site) and $\mathrm{Mo}^{6+}$ in the actual manganites phase. The deficiency in $2+$ will create $\mathrm{Mn}^{4+}$ ions and the overall $\mathrm{Mn}^{4+}$ ions are not changed. According to the charge balance the effect of Mo deficiency in the Mn site is negligible. On the other hand, the ionic radii of $2+$ is large compared with Mn ions, which will have a greater influence on lattice parameters rather than that of the magnetic and transport properties. A smaller suppression rate and long-range FM order in doped samples support the opening of another DE channel between $\mathrm{Mn}^{2+}-\mathrm{O}-\mathrm{Mn}^{3+}$ ions. This is further supported by effective magnetic moments deduced from the Curie-Weiss equation.

Fits of the magnetic susceptibility to a Curie-Weiss law above $T_{\mathrm{C}}$ yield a high effective Bohr magnetron number $P_{\text {eff }}$. The obtained $P_{\text {eff }}$ values are 5.57, 6.05, 6.43, 6.61, 6.81 and $6.92 \mu_{\mathrm{B}}$ for the samples with $x=0,0.01,0.02,0.03,0.04$ and 0.05 , respectively. According to a mean field approximation, the expected $P_{\mathrm{eff}}^{\mathrm{th}}$ can be calculated as $4.59,4.58,4.57,4.56$, 4.55 and $4.54 \mu_{\mathrm{B}}$ for the samples with $x=0,0.01,0.02,0.03$, 0.04 and 0.05 , respectively. The value of $P_{\text {eff }}^{\text {th }}$ is expected for a mixture of $(x) \mathrm{Mn}^{2+},(0.7) \mathrm{Mn}^{3+}$ and $(0.3-2 x) \mathrm{Mn}^{4+}$, where the average magnetic ion spin is taken as a weighted mean value of $S=5 / 2,2$ and $3 / 2$, respectively. The experimentally obtained value of $P_{\text {eff }}$ is higher than the $P_{\text {eff }}^{\text {th }}$ value and increases with $x$. This implies that the formation of FM spin clusters in the PM state [8] and the observed enhancement of $P_{\text {eff }}$ with Mo doping also supports the existence of $\mathrm{Mn}^{2+}$.

The temperature dependence of the resistivity $\rho(T)$ curves for the $\mathrm{La}_{0.7} \mathrm{Ca}_{0.15} \mathrm{Sr}_{0.15} \mathrm{Mn}_{1-x} \mathrm{Mo}_{x} \mathrm{O}_{3}$ samples measured at zero field are plotted in figure 5. The $\rho(T)$ curve of sample $x=0$ shows a single metal insulator transition $T_{\mathrm{MI}}$ at $328 \mathrm{~K}$, which coincide with $T_{\mathrm{C}}$. The value of $T_{\mathrm{MI}}$ decreases monotonically with increasing $x$, where $T_{\mathrm{MI}}$ of $\sim 316 \mathrm{~K}$ is 
observed for the sample with $x=0.05$. The suppression rate $\left(\mathrm{d} T_{\mathrm{MI}} / \mathrm{d} x\right)$ is found to be $2.4 \mathrm{~K}$ at $\%^{-1}$, which is smaller than that of the $\mathrm{d} T_{\mathrm{C}} / \mathrm{d} x$. Applying the magnetic field $H$ lowers the resistivity (not shown here) for all the samples and leads to an increase in $T_{\mathrm{MI}}$ and a negative CMR effect. The temperature of $T_{\mathrm{MI}}$ is higher than that of the $T_{\mathrm{C}}$ for the Mo-doped samples (shown in the phase diagram), which is rarely found even in ideal single crystals. This anomalous behaviour and the evidence of magnetic spin clusters above $T_{\mathrm{C}}$ supports the existence of the GP in Mo-doped samples.

Griffiths [10] considered a system with randomly distributed spins, in which a fraction $p$ of the lattice sites were occupied by magnetic ions and the rest remained vacant. As $p=1$, the magnetic ions occupy every lattice site, and the long-range FM transition occurs at a temperature $T_{\mathrm{G}}$ called the Griffiths temperature. For a real system, $p<1$, the transition is suppressed to a lower temperature $T_{\mathrm{C}}$. Bray [11] extended this argument to all systems in which disorder suppresses the magnetic transition temperature from $T_{\mathrm{G}}$ to $T_{\mathrm{C}}$. Recently, the Griffiths theory has been used to quantitatively explain the magnetic and transport properties of manganites [9,12-17]. Among the effects that characterize the GP are a random distribution of FM clusters for the temperature range $T_{\mathrm{C}}<T<T_{\mathrm{G}}$. On the other hand, the DE mechanism predicts that these magnetic clusters themselves are metallic due to the FM ordering of Mn spins inside the clusters. The FMM and PMI regions co-exist for the temperature range $T_{\mathrm{C}}<T<T_{\mathrm{G}}$, both randomly fill the space of the sample, predicted by the Griffiths theory incorporating the DE mechanism. Thus, the total resistivity of the system comprises both $\rho_{\mathrm{FM}}$ and $\rho_{\mathrm{PM}}$. Hence the total resistivity of the system can be expressed as $[48,49]$

$$
\rho=p \rho_{\mathrm{FM}}+(1-p) \rho_{\mathrm{PM}},
$$

where $p$ and $(1-p)$ are the volume fractions of the FMM and PMI regions, respectively. The volume fraction, $p$, of FMM clusters increases as the temperature is lowered from $T_{\mathrm{G}}$. Above $T_{\mathrm{G}}$, no FMM clusters exist and the conductivity is insulating, whereas below $T_{\mathrm{G}}$, the clusters of metallic conductivity grow upon cooling. Therefore, a temperature dependent $p$ is required to fit the experimental data. As the $p$ is increased to a critical value $p_{\mathrm{c}}$, the percolation threshold, the metallic percolation paths start to form. This is expected to occur around $T_{\mathrm{C}}$. The temperature dependence of $p$ were assumed to obey a simple two-energy level Boltzmann distribution and given as $p=1 /\left[1+\exp \left(-\Delta U / k_{\mathrm{B}} T\right)\right], \Delta U$ is the energy difference between the FMM and the PMI states, and is expressed as $\Delta U \sim-U_{0}\left(1-T / T_{\mathrm{C}}^{\bmod }\right), U_{0}$ is the energy difference for temperature well below $T_{\mathrm{C}}^{\text {mod }}[48,49]$.

In the FMM region $\left(T<T_{\mathrm{MI}}\right)$, to obtain a satisfactory fit of the experimental data in manganites, several mechanisms have been proposed. These include the different combinations of the electron-electron $\left(T^{2}\right)[50,51]$ with $T^{2.5}$ (combination of electron-electron, electron-phonon and electron-magnon [52, 53], single magnon $\left(T^{3}\right)$ [54], two magnon $\left(T^{4.5}\right)$ [55], spin-waves $\left(T^{3.5}\right)$ [56], and phonons $\left(T^{5}\right)$ [57] scatterings. Using the above combinations, the coefficient of the $T^{2}$ term in our samples was found to be $\sim 2.2 \times 10^{-7} \Omega \mathrm{cm} \mathrm{K}^{-2}$, which is too large to account for electron-electron interactions.

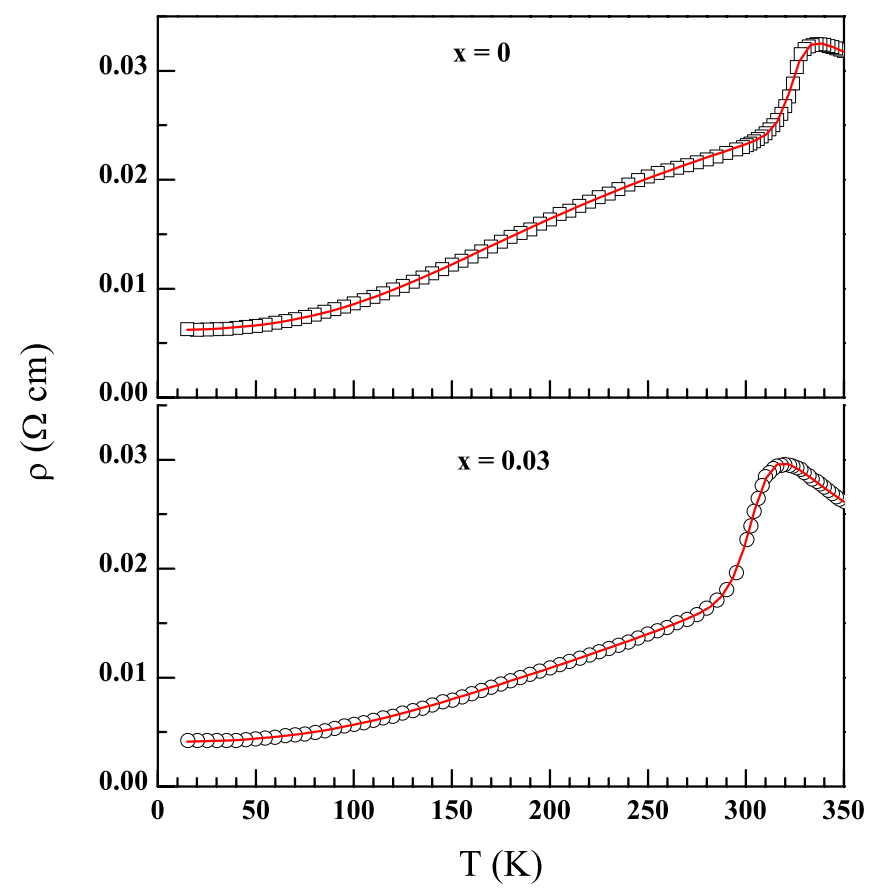

Figure 6. The temperature dependence of resistivity $\rho(T)$ curves for $x=0$ and $x=0.03$ samples measured at zero field. The solid lines represent fits to the percolation model.

According to Jaime et al [51], it should be $\sim 9 \times$ $10^{-9} \Omega \mathrm{cm} \mathrm{K}^{-2}$. Alexandrow and Bratkovsky [58] argued that even below $T_{\mathrm{C}}$, polaronic transport remains the dominant charge carrier. The impurity and two magnon scattering contributions included in polaronic conduction and the low temperature resistivity is governed by [59]

$$
\rho_{\mathrm{FM}}(T)=\rho_{0} \rho_{45} T^{4.5}+C / \sinh ^{2}\left(\hbar \omega_{\mathrm{s}} / 2 k_{\mathrm{B}} T\right),
$$

where $\rho_{0}$ is the electrical resistivity due to the grain boundary, domain boundary and other temperature independent processes, $\rho_{4.5}$ represents the electrical resistivity due to the two magnon scattering in the FMM phase, $C$ is a constant proportional to the effective mass of polarons, i.e. the electronphonon coupling strength, $\omega_{\mathrm{S}}$ is the average frequency of the softest optical mode. In our samples both including the $T^{4.5}$ term and without the 2 nd term data is not satisfied. However, the $T^{2.5}$ term in the empirical fit to the data expected to be significant in this system. So, the $\rho_{\mathrm{FM}}$ was analysed using polaronic conduction including impurity and $T^{2.5}$ terms $\rho_{\mathrm{FM}}(T)=\rho_{0}+\rho_{2.5} T^{2.5}+C / \sinh ^{2}\left(\hbar \omega_{\mathrm{s}} / 2 k_{\mathrm{B}} T\right)$ and in the high temperature range $\left(\rho_{\mathrm{PM}}\right)$ the adiabatic polaron model $[60,61]$ $\rho_{\mathrm{PM}}=\rho_{\alpha} T \exp \left(E_{\mathrm{a}} / k_{\mathrm{B}} T\right)$ was used. Equation (1) is used to examine our resistivity data over the whole temperature range. The solid lines in figure 6 show excellent agreement between the measured and calculated data using equation (1) for all the samples. From the non-linear fittings (figure 6), we estimated the volume fraction of the FMM (percolation threshold, $p$ ).

The typical temperature derivative of the volume fraction $p$ obtained by fitting the data to the percolation model is plotted in figure 7 along with the temperature dependence of the inverse susceptibility for $(a) x=0$ and $(b) x=0.03$. If the FM clusters have larger spins, $\chi(T)$ would show a large deviation 


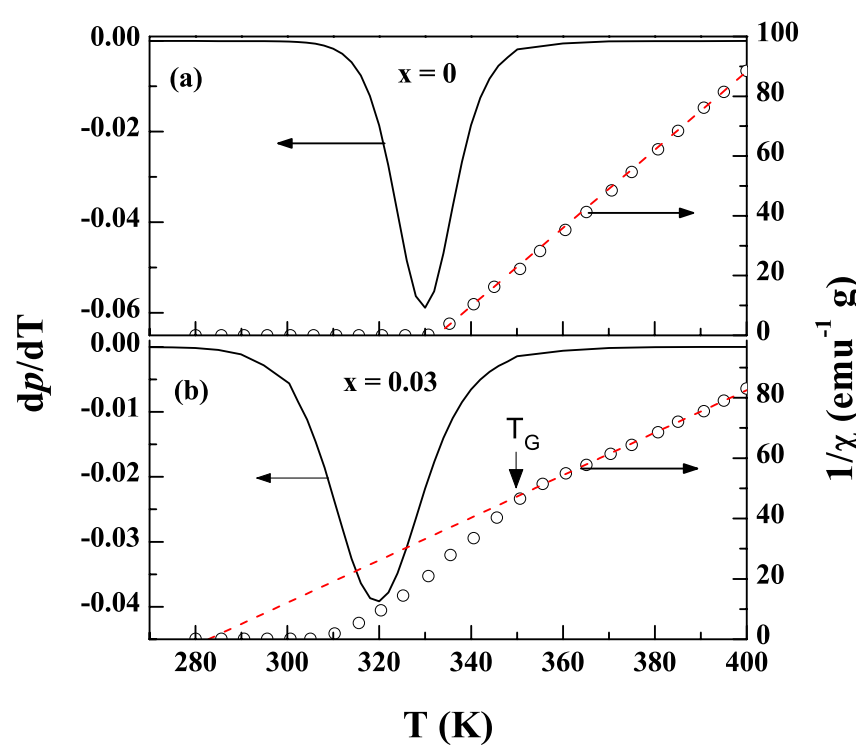

Figure 7. Temperature derivative of metallic volume fraction $p$ (solid lines) and the inverse of magnetic susceptibility as a function of temperature for the samples with $(a) x=0$ and $(b) 0.3$. The dashed lines are fitted curves according to the Curie-Weiss law.

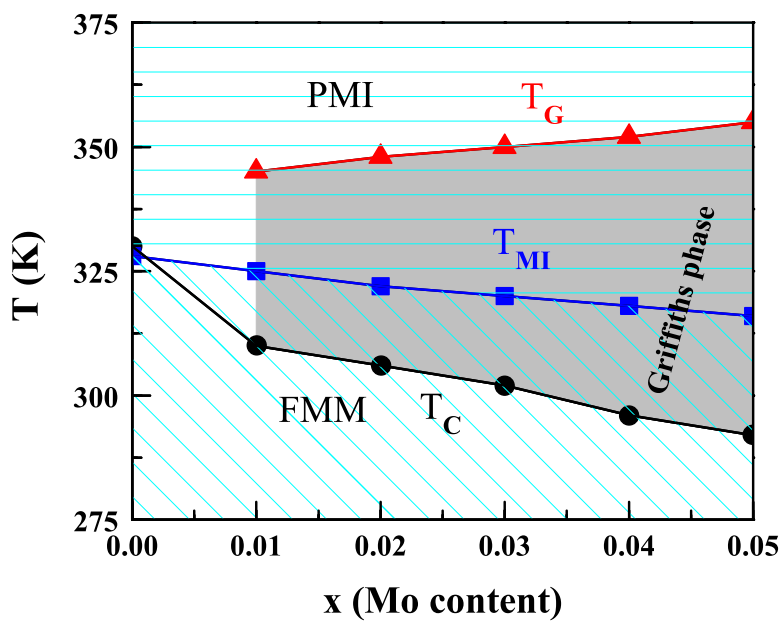

Figure 8. Transport and magnetic phase diagram for the $\mathrm{La}_{07} \mathrm{Ca}_{0.15}$ $\mathrm{Sr}_{0.15} \mathrm{Mn}_{1-x} \mathrm{Mo}_{x} \mathrm{O}_{3}$ system. The identified phases are PMI, FMM and GP (grey region).

from the Curie-Weiss form when the $T_{\mathrm{G}}$ appears. Therefore, the low field susceptibility would give a direct support for the existence of the GP. It is interesting to note that the undoped sample (figure 7(a)) shows no deviation from the Curie-Weiss law in the PM region and the onset of metallicity coincides with $T_{\mathrm{C}}$. But a significant downturn in the $\chi^{-1}-T$ curve at $T_{\mathrm{G}}$ well above $T_{\mathrm{C}}$ and the onset of metallicity do not coincide with $T_{\mathrm{C}}$ observed in the Mo doped samples (figure 7(b)). This suggests that more high spin FM clusters have embedded into the PM matrix before the PM-FM transition in the doped samples. The Mo substitution induces an inhomogeneous distribution of magnetic clusters.

Figure 8 represents a proposed transport and magnetic phase diagram for the $\mathrm{La}_{0.7} \mathrm{Ca}_{0.15} \mathrm{Sr}_{0.15} \mathrm{Mn}_{1-x} \mathrm{Mo}_{x} \mathrm{O}_{3}$ system showing the effects of increasing disorder by substitution of Mo at the $\mathrm{Mn}$ site. For the $x=0$ sample only one phase

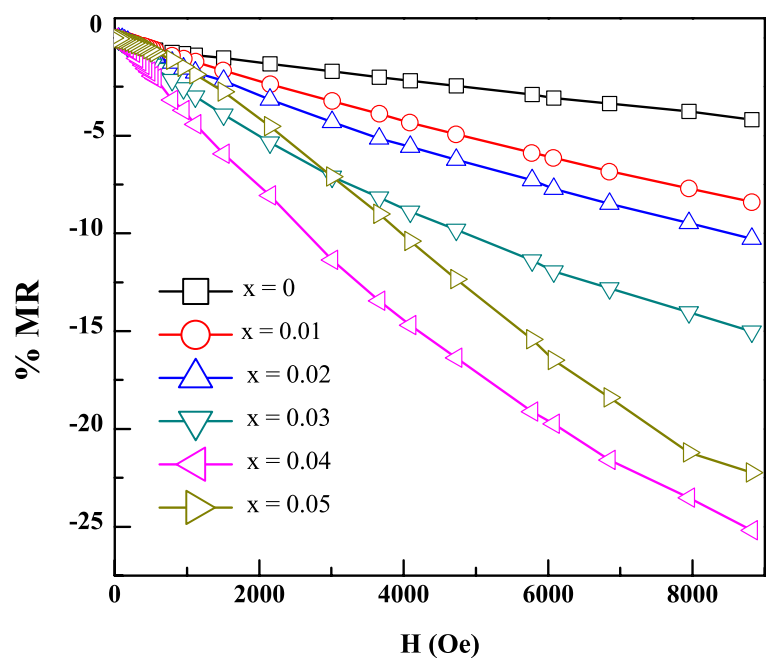

Figure 9. The field dependence of MR ratio measured at room temperature for the $\mathrm{La}_{0.7} \mathrm{Ca}_{0.15} \mathrm{Sr}_{0.15} \mathrm{Mn}_{1-x} \mathrm{Mo}_{x} \mathrm{O}_{3}$ samples.

change occurs i.e. PMI to FMM. But in the Mo-doped samples, different phases co-exist: PMI, GP (grey region) and FMM. A special region, corresponding to the temperature range of $T_{\mathrm{C}}<T<T_{\mathrm{G}}$, is indicated by the grey region (GP). It is seen that GP is enlarged with increasing Mo content. This is due to the enhanced localization of carriers because of the increasing $\mathrm{Mn}^{2+}$ induced by $\mathrm{Mo}^{6+}$ ions resulting in random charge trapping centers at the Mn site. Inside this region, the samples show complicated conductivity and anomalous PM behaviour simultaneously, the Griffiths theory incorporating the DE mechanism is applied to the present system.

The field dependence of the MR ratio measured at room temperature for the $\mathrm{La}_{0.7} \mathrm{Ca}_{0.15} \mathrm{Sr}_{0.15} \mathrm{Mn}_{1-x} \mathrm{Mo}_{x} \mathrm{O}_{3}$ system is plotted in figure 9. The MR versus $H$ curves reveal the typical features of the intrinsic CMR behaviour. The MR ratio near room temperature increases apparently with the increasing Mo-doping $x$. As discussed earlier, the Mo doping leads to the formation of FMM and PMI clusters. The MR is governed by the field induced enhancement in the size and connectivity of these FMM clusters. When a magnetic field is applied, the size of the PMI clusters remains unaltered while that of the FMM ones increase, leading to a decrease in resistivity. For $x=0.04$, MR reaches a maximum value of $25.2 \%$ at room temperature, which is six times higher than that of the undoped sample for $H=8.9 \mathrm{kOe}$. This suggests that at this concentration the applied magnetic field is strong enough to induce optimum density of the conduction channels. However, for the $x=0.05$ sample the density of the PMI clusters is large due to the impurity phase, and consequently the MR is relatively smaller. As present, the Mo substituted samples comprise dual DE interactions having $\mathrm{Mn}^{2+}-\mathrm{Mn}^{3+}$ and $\mathrm{Mn}^{3+}-$ $\mathrm{Mn}^{4+}$ along with the resulting three superexchange (SE) interactions between $\mathrm{Mn}^{3+}-\mathrm{Mn}^{3+}, \mathrm{Mn}^{4+}-\mathrm{Mn}^{4+}$ and $\mathrm{Mn}^{2+}-$ $\mathrm{Mn}^{2+}$. These competing DE and SE interactions along with the inhomogeneity arise due to differences in the Mn size and valences are expected to enhance the MR as compared with the single $\mathrm{DE}$ of $\mathrm{Mn}^{3+}-\mathrm{Mn}^{4+}$. 


\section{Conclusions}

We have investigated the influence of Mo substitution at the Mn site on structure, magnetic and magneto transport properties in the $\mathrm{La}_{0.7} \mathrm{Ca}_{0.15} \mathrm{Sr}_{0.15} \mathrm{Mn}_{1-x} \mathrm{Mo}_{x} \mathrm{O}_{3}$ system. Powder $\mathrm{x}$-ray analysis reveals that the structural change rhombohedral to orthorhombic occurs with Mo doping. The $M(T)$ and $\rho(T)$ data show an anomalous PM behaviour and complicated conductivity simultaneously in the temperature range $T_{\mathrm{C}}<$ $T<T_{\mathrm{G}}$ for the Mo-doped samples. In this range the results are discussed within the framework of the Griffiths theory incorporating the DE mechanism. The MR ratio near room temperature increases apparently with increasing $x$. The Mn-site disorder and the existence of FM coupling between $\mathrm{Mn}^{2+}-\mathrm{O}-\mathrm{Mn}^{3+}$ due to the $\mathrm{Mn}^{2+}$ ions induced by $\mathrm{Mo}^{6+}$ in the samples are responsible for the results above. These results suggest that Mn site Mo substitution could be a potential way to tune CMR at room temperature.

\section{Acknowledgments}

This work is supported by National Taiwan University's ‘Aim for Top University project (ATU)', under Grant No 95R004, and by the National Science Council of ROC under Grant No NSC95-2112-M-002-049-MY3.

\section{References}

[1] von Helmolt R, Wecker J, Holzapfel B, Schultz L and Samwer K 1993 Phys. Rev. Lett. 712331

[2] Imada M, Fujimori A and Tokura Y 1998 Rev. Mod. Phys. 701039

[3] Rao C N R and Raveau B (ed) 1998 Colossal Magneto Resistance, Charge Ordering, and Related Properties of Manganese Oxides (Singapore: World Scientific)

Tokura Y (ed) 2000 Colossal Magnetoresistive Materials (New York: Gordon and Breach)

[4] Zener C 1951 Phys. Rev. 82403 de Gennes P G 1960 Phys. Rev. 118141

[5] Millis A J, Littlewood P B and Shraiman B I 1995 Phys. Rev. Lett. 745144

[6] Dagotto E (ed) 2002 Nanoscale Phase Separation and Colossal Magnetoresistance (Berlin: Springer)

[7] Uehara M, Mori S, Chen C H and Cheong S-W 1999 Nature 399560

[8] De Teresa J M, Ibarra M R, Algarabel P A, Ritter C, Marquina C, Blasco J, Garcia J, del Moral A and Arnold Z 1997 Nature 386256

[9] Burgy J, Mayr M, Martin-Mayor V, Moreo A and Dagotto E 2001 Phys. Rev. Lett. 87277202

[10] Griffiths R B 1969 Phys. Rev. Lett. 2317

[11] Bray A J 1987 Phys. Rev. Lett. 59586

[12] Salamon M B and Chun S H 2003 Phys. Rev. B 68014411

[13] Salamon M B, Lin P and Chun S H 2002 Phys. Rev. Lett. 88197203

[14] Rama N, Ramachandra Rao M S, Sankaranarayanan V, Majewski P, Gepraegs S, Opel M and Gross R 2004 Phys. Rev. B 70224424

[15] Deisenhofer J, Braak D, Krug von Nidda H-A, Hemberger J, Eremina R M, Ivanshin V A, Balbashov A M, Jug G, Loidl A, Kimura T and Tokura Y 2005 Phys. Rev. Lett. 95257202

[16] Chan P Y, Goldenfeld N and Salamon 2006 Phys. Rev. Lett. 97137201
[17] Jiang W, Zhou X, Williams G, Mokovskii Y and Glazyrin K 2008 Phys. Rev. B 77064424

[18] Tong P, Kim B, Kwon D, Qian T, Lee Sung-IK, Cheong S-W and Kim B G 2008 Phys. Rev. B 77184432

[19] Jiang W, Zhou X, Williams G, Mokovskii Y and Glazyrin K 2007 Phys. Rev. Lett. 99177203

[20] Blasco J, García J, de Teresa J M, Ibarra M R, Perez J, Algarabel P A, Marquina C and Ritter C 1997 Phys. Rev. B 558905

[21] Nam D N H, Dai N V, Thanh T D, Tuong L T C, Hong L V, Phuc N X, Hong H S and Khien N V 2008 Phys. Rev. B 77224420

[22] Wang L M, Lai J H, Wu J L, Kuo Y-K and Chang C L 2000 J. Appl. Phys. 875597

[23] Sun Y, Xu X, Zheng L and Zhang Y 1999 Phys. Rev. B 6012317

[24] Huang Y H, Liao C S, Wang Z M, Li X H, Yan C H, Sun J R and Shen B G 2002 Phys. Rev. B 65184423

[25] Lakshmi L S, Dörr K, Nenkov K, Handstein A, Müller K-H and Sastry V S 2007 J. Phys.: Condens. Matter 19216218

[26] Seetha Lakshmi L, Sridharan V, Sukumar A A, Kamruddin M, Sastry V S and Raju V S 2006 J. Phys.: Condens. Matter 184427

[27] Sun Y, Xu X and Zhang Y 2000 Phys. Rev. B 63054404

[28] Rivadulla F, Lopez-Quintela M A, Hueso L E, Sande P, Rivas J and Sanchez R D 2000 Phys. Rev. B 625678

[29] Gayathri N, Raychaudhuri A K, Tiwary S K, Gundakaram R, Arulraj A and Rao C N R 1997 Phys. Rev. B 561345

[30] Wang K Y, Song W H, Dai J M, Ye S L, Wang S G, Fang J, Chen J L, Gao B J, Du J J and Sun Y P 2001 J. Appl. Phys. 906263

[31] Ahn K H, Wu X W, Liu K and Chien C L 1996 Phys. Rev. B 5415299

[32] Ghosh K, Ogale S B, Ramesh R, Greene R L, Venkatesan T, Gapchup K M, Bathe R and Patil S I 1999 Phys. Rev. B 59533

[33] Kimura T, Tomioka Y, Kumai R, Okimoto Y and Tokura Y 1999 Phys. Rev. Lett. 833940

[34] Moritomo Y, Machida A, Mori S, Yamamoto N and Nakamura A 1999 Phys. Rev. B 609220

[35] Machida A, Moritomo Y, Ohoyama K, Katsufuji T and Nakamura A 2002 Phys. Rev. B 65064435

[36] Hébert S, Martin C, Maignan A, Retoux R, Hervieu M, Nguyen N and Raveau B 2002 Phys. Rev. B 65104420

[37] Lu W J, Sun Y P, Zhao B C, Zhu X B and Song W H 2006 Phys. Rev. B 73174425

[38] Raveau B, Zhao Y M, Martin C, Hervieu M and Maignan A 2000 J. Solid State Chem. 149203

[39] Kundaliya D C, Vij R, Kulkarni R G, Varughese B, Nigam A K and Malik S K 2005 J. Appl. Phys. 98013905

[40] Narsinga Rao G and Chen J W 2007 Effect of Mo doping for the $\mathrm{Mn}$ site in the ferromagnetic manganite $\mathrm{La}_{0.7} \mathrm{Ca}_{0.3} \mathrm{MnO}_{3}$ Preprint arXiv:0705.3080

[41] Hwang H Y, Cheong S-W, Radaelli P G, Marezio M and Batlogg B 1995 Phys. Rev. Lett. 75914

[42] Radaelli P G, Marezio M, Hwang H Y, Cheong S-W and Batlogg B 1996 Phys. Rev. B 548992

[43] Tomioka Y, Asamitsu A and Tokura Y 2000 Phys. Rev. B 63024421

[44] Mira J, Rivas J, Hueso L E, Rivadulla F, Lopez Quintela M A, Senaris Rodriguez M A and Ramos C A 2001 Phys. Rev. B $\mathbf{6 5} 024418$

[45] Ulyanov A N, Yu S C, Yu Starostyuk N, Pismenova N E, Moon Y M and Lee K W 2002 J. Appl. Phys. 908900

[46] Pfoertsch D E and McCarthy G J 1978 Powder Diffraction Files 30-1287

[47] Shannon R D and Prewitt C T 1976 Acta Crystallogr. Sect. A Cryst. Phys., Diffr., Theor. Gen. Crystallogr. 32785 
[48] Yuan S L, Zhao W Y, Zhang G Q, Tu F, Peng G, Liu J, Yang Y P, Li G, Jiang Y, Zeng X Y, Tang C Q and Jin S Z 2000 Appl. Phys. Lett. 774398

[49] Yuan S L, Li Z Y, Peng G, Xiong C S, Xiong Y H and Tang C Q 2001 Appl. Phys. Lett. 7990

[50] Snyder G J, Hiskes R, DiCarolis S, Beasley M R and Geballe T H 1996 Phys. Rev. B 5314434

[51] Jaime M, Lin P, Salamon M B and Han P D 1998 Phys. Rev. B 58 R5901

[52] Schiffer P, Ramirez A P, Bao W and Cheong S-W 1995 Phys. Rev. Lett. 753336

[53] Marcone S, Perroni C A, Cataudella V, Adamo C, Angeloni M, Aruta C, De Filippis G, Miletto F, Orpallo A, Perna P, Yu Petrov A, di Uccio U S and Maritato L 2005 Phys. Rev. B 7164415
[54] Akimoto T, Moritomo Y, Nakamura A and Furukawa N 2000 Phys. Rev. Lett. 853914

[55] Kubo K and Ohata N 1972 J. Phys. Soc. Japan 3321

[56] Chen K J, Habermeier H-U, Zhang C L, Zhang H and Almasara C C 2003 Phys. Rev. B 67134405

[57] Li G, Zhou H-D, Feng S J, Fan X-J, Li X-G and Wang Z D 2002 J. Appl. Phys. 921406

[58] Alexandrov A S and Bratkovsky A M 1999 Phys. Rev. Lett. 82141

[59] Zhao G, Keller H and Prellier W 2000 J. Phys.: Condens. Matter 12 L361

[60] Mott N F 1990 Metal-Insulator Transitions (London: Taylor and Francis)

[61] Banerjee A, Pal S and Chaudhuri B K 2001 J. Chem. Phys. 1151550 